\title{
Exposición a tabaco, alcohol y drogas de abuso en gestantes. Estudio de prevalencia en gestantes de Málaga (España)
}

\author{
Exposure to tobacco, alcohol and drugs of abuse \\ during pregnancy. A study of prevalence among \\ pregnant women in Malaga (Spain)
}

\author{
Marta Blasco-Alonso*, Ernesto González-Mesa*, Milagros Gálvez Montes*, Isabel lozano \\ Bravo*, Federico Merino Galdón*, Francisco Cuenca Campos*, Gema Marín Schiaffino*, Sergio \\ Pérez Torres*, José Herrera Peral*, Inmaculada Bellido Estévez** \\ * Departamento de Obstetricia y Ginecología. Universidad de Málaga. Hospital Regional Universitario de Málaga. \\ ** Departamento de Farmacología. Facultad de Medicina. Universidad de Málaga.
}

\section{Resumen}

La prevalencia de hábitos tóxicos en la población de mujeres que quedan embarazadas es similar a la de la población general, por lo que la exposición fetal a tóxicos es elevada en el período de mayor vulnerabilidad, sobre todo en relación al neurodesarrollo y la organogénesis. El presente estudio ha sido desarrollado para conocer el nivel de exposición prenatal a tabaco, alcohol u otras drogas en la ciudad de Málaga (España). El trabajo responde a un diseño observacional de corte transversal sobre el consumo de tóxicos durante el embarazo, y se basa en la autodeclaración de las gestantes mediante la cumplimentación de un cuestionario. Se reclutaron 451 gestantes de primer, segundo y tercer trimestre. La prevalencia de consumo en cada uno de los trimestres resultó ser respectivamente del $21.2 \%, 18.5 \%$ y $13.3 \%$ para el tabaco, $40.7 \%, 23.1 \%$ y $17.1 \%$ para el alcohol y del $4.8 \%, 1.9 \%$ y $1.2 \%$ para cannabis. En los tres trimestres, un mayor nivel de estudios se asoció a un menor consumo de tabaco (RR 0,659 [0.537-0.810] p< 0.0001) y una mayor exposición al alcohol (RR 1.87 [1.30-2.69] $\mathrm{p}<0.0007)$. Los resultados obtenidos, sobre todo en relación al consumo de alcohol, son suficientemente llamativos como para alertar a los proveedores de atención obstétrica sobre la necesidad de poner en marcha medidas preventivas.

Palabras clave: Exposición prenatal, Consumo materno de alcohol, Tabaquismo materno, Prevalencia, Drogas ilícitas durante el embarazo, Alto riesgo obstétrico.

\begin{abstract}
The prevalence of substance abuse in women who become pregnant is similar to that of the general population, resulting in a high fetal exposure rate during the most vulnerable period regarding neurodevelopment and organogenesis. The present study was intended to assess the level of prenatal exposure to tobacco, alcohol or illicit drugs in the city of Málaga (Spain). It was designed as a cross-sectional study, and based on the anonymous self-reports of participants. A total of 451 pregnant women were recruited in the first, second or third trimester. The prevalence in each of the quarters respectively was $21.2 \%, 18.5 \%$ and $13.3 \%$ for smoking, $40.7 \%, 23.1 \%$ and $17.1 \%$ for alcohol and $4.8 \%, 1.9 \%$ and $1.2 \%$ for cannabis. We also found that a higher educational level was associated with a lower consumption of tobacco (RR 0.659 [0.537-0.810] $\mathrm{p}<0.0001$ ) and greater exposure to alcohol (RR 1.87 [1.30-2.69] $\mathrm{p}<0.0007)$. These results, particularly in regard to alcohol intake, are sufficiently alarming to alert obstetric care providers about the need to implement preventive measures. Key words: Prenatal exposure, Maternal alcohol intake, Maternal smoking, Prevalence, Drug abuse during pregnancy, High risk pregnancy.
\end{abstract}


S egún datos del Plan Nacional sobre Drogas (Delegación del Gobierno para el Plan Nacional sobre Drogas, 2011) obtenidos mediante encuesta domiciliaria a la población española entre 15 y 64 años el $35.1 \%$ de los encuestados consumían diariamente tabaco y el $15.3 \%$ alcohol. Un $6.5 \%$ de la población encuestada admitía haber consumido cannabis en los últimos 30 días, cocaína el 1.4\% y anfetaminas el $0.6 \%$. Datos recogidos en la Encuesta Nacional de Salud 2012 ponen de manifiesto que el $61.4 \%$ de las mujeres en edad fértil consumieron alcohol en el año previo (Ministerio de Sanidad, Servicios Sociales e Igualdad, 2013).

La prevalencia de hábitos tóxicos en la población de mujeres que quedan gestantes es similar a la de la población general, por lo que la exposición fetal a tóxicos es elevada durante al menos el inicio del primer trimestre, existiendo al final del embarazo aún un grupo de pacientes que mantiene un nivel importante de exposición (Cnattingius, 2004).

Aunque resulta muy difícil estimar todas las consecuencias del consumo de estas sustancias durante el embarazo, sabemos al menos que los efectos en el feto dependen fundamentalmente del momento y la intensidad de la exposición, siendo el primer trimestre el periodo de mayor vulnerabilidad sobre todo en relación al neurodesarrollo y la organogénesis. El consumo de tóxicos se ha asociado además a retraso del crecimiento intrauterino, desprendimiento de placenta, parto prematuro (Bernstein, Plociennik, Stahle, Badger y Secker-Walker, 2000; Bouras et al., 2013), aborto espontáneo, embarazo ectópico, placenta previa y los síndromes de distrés respiratorio y muerte súbita infantil (Comité Nacional para la Prevención del Tabaquismo, 2007; Genbacev, Blass, Joslin y Fisher, 1995; Toothily, Stewart, Coles, Andrews y Cartlidge, 1999).

No existe demasiada información en nuestro país sobre la prevalencia del consumo de tóxicos durante el embarazo. Los estudios sobre el consumo de tabaco ponen de manifiesto una prevalencia que oscila entre el 27\% (Doz-Mora et al., 2002) y el $34 \%$ de las gestantes a término (Pichini et al., 2002). Por otro lado, Martínez-Frías, Rodríguez-Pinilla y Bermejo (2005) observan una tendencia al incremento del número de mujeres que fuman durante el embarazo desde 1975, con una prevalencia entre 1995 y 2002 del $30 \%$. Con relación al consumo de alcohol, la información procedente del grupo Eurocare (2011) indica que en nuestro país, el $25 \%$ de las mujeres consume alcohol durante el embarazo, habiéndose descrito una incidencia de síndrome alcohólico fetal de 0.46 por diez mil nacimientos (Eurocat, 2014). No existen datos nacionales sobre prevalencia de los trastornos del espectro alcohólico fetal, si bien en otros países llegan a ser hasta 10 veces más frecuente. Los datos sobre consumo de drogas ilícitas durante el embarazo son escasos. En un estudio realizado en Barcelona sobre muestras de meconio se observó que un $5.3 \%$ eran positivas a cannabis, un $4.7 \%$ a heroína y un $2.6 \%$ a cocaína (García-Algar et al., 2009).
El presente estudio ha sido diseñado con objeto de conocer el nivel de exposición prenatal a tabaco, alcohol $\mathrm{u}$ otras drogas en la ciudad de Málaga y poder plantear estrategias de salud pública que se sumen a las existentes, y que permitan reducir el impacto negativo que el consumo de estas sustancias tiene durante el embarazo y sobre la salud de los recién nacidos. La sistematización de la asistencia obstétrica mediante el diseño de procesos asistenciales permite establecer intervenciones en este sentido. La guía clínica de atención al embarazo normal (Grupo de trabajo de la Guía de Práctica Clínica de Atención en el Embarazo y Puerperio, 2014), basándose en la evidencia científica, establece algunas recomendaciones y sugerencias sobre la detección de conductas de riesgo relacionadas con el consumo de sustancias tóxicas, si bien, la integración de dichas recomendaciones en la práctica asistencial depende de factores locales relacionados con el contexto organizativo y con la motivación del personal implicado en la asistencia, no existiendo datos sobre su cumplimiento a nivel nacional. La eficacia de la intervención educativa en la reducción del consumo durante el embarazo, ya ha sido evaluada en estudios anteriores (Chang, Wilkins-Haug, Berman y Goetz, 1999; O'Connor y Whaley, 2007; Reynolds, Coombs, Lowe, Peterson y Gayoso, 1995), si bien ninguno de estos estudios ha sido realizado en nuestro país.

Nuestro estudio se sitúa en la base de una línea de trabajo en salud reproductiva encaminada a evitar la exposición de las gestantes al tabaco, alcohol y otras drogas.

\section{Método}

Para estudiar la prevalencia de exposición a tabaco, alcohol u otras drogas de abuso de las gestantes en Málaga se diseñó un estudio observacional, descriptivo, transversal de prevalencia. Antes de la puesta en marcha del estudio se obtuvo la correspondiente autorización del Comité de Ética e Investigación de Málaga-Nordeste

\section{Participantes}

La población de estudio estuvo constituida por las gestantes que acudieron a control obstétrico a nuestro hospital, durante los meses de noviembre y diciembre de 2013.

Nuestra maternidad representa el centro de referencia para la atención especializada obstétrica y ginecológica de 14 centros de atención primaria del distrito sanitario Málaga, con más de 5000 partos atendidos durante 2013. Aunque existen otros centros con actividad obstétrica en nuestra capital, el volumen de actividad hace que la población atendida en nuestro servicio sea altamente representativa de la población general de gestantes de la ciudad.

En la organización de la asistencia obstétrica en Andalucía, está previsto que las gestantes con embarazo de bajo riesgo mantengan el control de la gestación en atención primaria acudiendo al hospital una vez en cada trimestre para 
la evaluación clínica y ecográfica por el médico especialista en obstetricia y ginecología. Concretamente las visitas están programadas para las semanas 12, 20 y 32 .

La población de estudio quedó constituida por las pacientes que acudían a la consulta de control obstétrico en la semana 12, 20 o 32. Considerando una prevalencia empírica (Cnattingius, 2004) de tabaquismo entre el 20 y el $40 \%$ y una frecuencia de consumo de alcohol (García-Algar et al., 2008) en embarazadas hasta del $45 \%$ estimamos con un nivel de confianza del $95 \%$ y una precisión del $5 \%$ que sería necesario reclutar un mínimo de 350 gestantes para obtener una muestra representativa.

Se realizó un muestreo consecutivo de las gestantes que acudieron a control obstétrico a nuestra maternidad en el último trimestre de 2013, reclutándose finalmente para el estudio un total de 451 participantes. De ellas 184 se encontraban en el primer trimestre, 121 en el segundo y 146 en el tercero.

\section{Procedimiento}

Las pacientes que accedieron a participar en el estudio contestaron las preguntas que sobre el consumo de tabaco, alcohol y otras drogas fueron recogidas en un cuestionario de consumo. Junto al cuestionario se entregaba una hoja informativa de consentimiento, en la que además de justificar el desarrollo del estudio se insistía en el carácter anónimo de las respuestas. Las propias gestantes introducían personalmente el cuestionario cumplimentado en una caja precintada, de manera que no era posible establecer la trazabilidad de ningún cuestionario, ya que además carecían de datos identificativos. Con idea de salvaguardar el anonimato de las pacientes no se solicitó en ningún momento la firma de la paciente, entendiendo que el hecho de cumplimentar el cuestionario e introducirlo personalmente en la caja de recogida conllevaba implícitamente la aceptación para ser incluida en el estudio. Cualquier paciente podía negarse a participar antes, durante o tras la cumplimentación del cuestionario con límite en el momento de la introducción del mismo en la caja de recogida. No obstante, el número de gestantes que renunciaron a participar fue inferior al $3.2 \%$.

\section{Instrumentos}

Las gestantes contestaron un cuestionario (Anexo 1) autoadministrado y anónimo sobre el consumo de sustancias, basado en preguntas directas centradas en valorar la existencia de consumo, así como la frecuencia y la intensidad del mismo, adaptado, a su vez, de cuestionarios validados y utilizados con anterioridad en estudios sobre exposición prenatal a drogas de abuso durante la gestación (Gómez, Conde y Aguiar, 2001). Con relación al consumo de tabaco las pacientes debían contestar sobre el número de cigarrillos diarios o semanales que consumían, tanto en el momento de cumplimentar el cuestionario, como antes del embarazo, así como la edad en la que inició el consumo. Igualmente, se preguntaba sobre el consumo de cigarrillos por parte de su pareja. Para conocer los datos referentes al consumo de alcohol, las pacientes debían contestar sobre la existencia de consumo, cantidad absoluta ingerida (número de copas y tipo de bebida consumida) y frecuencia del mismo, con ítems incorporados del dominio sobre consumo de riesgo de alcohol del test AUDIT (Babor, Higgins-Biddle, Saunders y Monteiro, 2001). Con relación al consumo de otras drogas de abuso, las gestantes debían contestar sobre el consumo de cannabis, cocaína, éxtasis, metadona, heroína o anfetaminas y la frecuencia diaria, semanal, mensual u ocasional con la que consumían. El cuestionario incluía también preguntas referentes al contexto sociodemográfico y familiar de las gestantes, con relación a la edad, ocupación, nivel de estudios, paridad, reproducción asistida, nacionalidad, código postal del domicilio, consumo de tabaco, alcohol o drogas de abuso previo al embarazo y consumo de tabaco, alcohol o drogas de abuso por su pareja.

\section{Análisis de los datos}

Las variables independientes consideradas hacían referencia a aspectos sociodemográficas y de salud: edad de la gestante, trimestre de embarazo, nivel de estudios, profesión, paridad, uso de técnicas de reproducción asistida, nacionalidad, código postal, consumo de tabaco, alcohol o drogas de abuso previo al embarazo y consumo de tabaco, alcohol o drogas de abuso por su pareja.

Como principales variables de resultado se estudiaron el consumo de tabaco, el consumo de alcohol y el consumo de otras drogas de abuso. Las tres variables se codificaron como variables dicotómicas (consumo o ausencia de consumo). La información sobre frecuencia de consumo en los tres casos, quedó recogida como variables categóricas (consumo diario, semanal, mensual, ocasional y ausencia de consumo). Para estudiar la intensidad del consumo de tabaco se consideró como variable resultado el número de cigarrillos consumidos (rango de 0 a n).

Para estudiar la intensidad de exposición al alcohol se consideró el volumen de bebidas alcohólicas consumidas (vaso de chupito, vaso/copa grande, lata o botella), diariamente, semanalmente o mensualmente, según el patrón de consumo declarado de cada gestante, distinguiendo a su vez, entre consumo de cerveza, vino y destilados (solos o en combinación). Para la posterior codificación y análisis estadísticos se transformaron los volúmenes en unidades de bebida estándar de alcohol (UBE). La UBE hace referencia al contenido medio en alcohol de una consumición habitual atendiendo a su graduación y volumen. La UBE en nuestro país contiene 10 gramos de alcohol puro, habiéndose establecido la conversión entre volumen y UBE de la siguiente forma: una consumición estándar de cerveza y una consumición estándar de vino equivalen a 1 UBE, y una consumición estándar de destilados equivale a 2 UBE (Llopis, Gual y Rodríguez-Martos, 2000) . 
Finalmente, para estudiar el consumo de drogas de abuso se codificó una variable que recogía la frecuencia de consumo de cannabis, cocaína, éxtasis, metadona, heroína o anfetaminas, distinguiendo en los casos de consumo, entre patrón de consumo diario, semanal u ocasional. Inicialmente se realizó un análisis descriptivo de las variables de estudio. El análisis de las variables independientes permitió la estratificación de la muestra en función de sus diferentes categorías. Para analizar la existencia de posibles diferencias significativas en las frecuencias observadas entre los diferentes grupos de estratificación se aplicó el test de la Chi-cuadrado cuando se analizaron las variables dicotómicas sobre consumo de tabaco, alcohol u otras drogas. Para los casos de tablas bidimensionales hemos calculado los RR y sus IC al 95\%. Una vez comprobada la normalidad de la distribución, para analizar las diferencias existentes en los valores de las variables continuas (intensidad de consumo) se aplicó la prueba $\mathrm{T}$ de Student para dos muestras independientes o ANOVA de un factor cuando el número de categorías de la variable independiente era superior a dos. Los análisis estadísticos se realizaron utilizando el programa SPSS, versión 15.0 (SPSS Inc., Chicago, IL).

\section{Resultados}

Las principales características demográficas de las gestantes quedan recogidas en la TABLA 1 . La edad media observada fue de 31.4 años (DT 5.2), tratándose en el 55.16\% de

Tabla 1

Principales características sociodemográficas de la muestra

\begin{tabular}{|c|c|c|c|}
\hline Características & $\begin{array}{c}1^{\mathrm{er}} \text { Trimestre } \\
\text { ( } \mathrm{N}=184)\end{array}$ & $\begin{array}{c}2^{\circ} \text { Trimestre } \\
(\mathrm{N}=121)\end{array}$ & $\begin{array}{c}\mathbf{3}^{\varepsilon \rho} \text { Trimestre } \\
\text { (N = 146) }\end{array}$ \\
\hline \multicolumn{4}{|l|}{ Edad } \\
\hline Edad en años & 30.4 & 33.6 & 30.2 \\
\hline Primiparidad & $108(58.7 \%)$ & $62(51.2 \%)$ & $55(55.6 \%)$ \\
\hline \multicolumn{4}{|l|}{ Situación laboral } \\
\hline $\begin{array}{l}\text { No trabaja } \\
\text { En activo } \\
\text { Busca empleo }\end{array}$ & $\begin{array}{c}46(25.3 \%) \\
104(57.1 \%) \\
32(17.6 \%)\end{array}$ & $\begin{array}{c}26(21 \%) \\
77(63 \%) \\
18(14.5 \%)\end{array}$ & $\begin{array}{l}47(32.1 \%) \\
69(37.5 \%) \\
29(19.8 \%)\end{array}$ \\
\hline \multicolumn{4}{|l|}{ Nivel de estudios } \\
\hline $\begin{array}{l}\text { Sin estudios } \\
\text { Obligatorios } \\
\text { Bachil/Equiv } \\
\text { Universitarios }\end{array}$ & $\begin{array}{c}13(7.1 \%) \\
64(34.8 \%) \\
44(23.9 \%) \\
57(31 \%)\end{array}$ & $\begin{array}{c}4(3.3 \%) \\
29(24 \%) \\
39(32.2 \%) \\
47(38.8 \%)\end{array}$ & $\begin{array}{c}11(7.5 \%) \\
46(31.5 \%) \\
34(23.2 \%) \\
31(21.2 \%)\end{array}$ \\
\hline \multicolumn{4}{|l|}{ Nacionalidad } \\
\hline $\begin{array}{l}\text { España } \\
\text { Europa del Este } \\
\text { Sudamérica } \\
\text { China } \\
\text { África } \\
\text { Magreb }\end{array}$ & $\begin{array}{c}163(90.1 \%) \\
2(1.1 \%) \\
5(2.8 \%) \\
1(0.6 \%) \\
1(0.6 \%) \\
8(4.4 \%)\end{array}$ & $\begin{array}{c}113(95.0 \%) \\
- \\
2(1.7 \%) \\
- \\
- \\
2(1.6 \%)\end{array}$ & $\begin{array}{c}91(91.7 \%) \\
1(0.6 \%) \\
5(4.1 \%) \\
1(0.6 \%) \\
2(1.2 \%) \\
3(1.8 \%)\end{array}$ \\
\hline \multicolumn{4}{|l|}{ Repr. Asistida } \\
\hline $\begin{array}{l}\text { No } \\
\text { Sí }\end{array}$ & $\begin{array}{c}175(95.6 \%) \\
7(3.8 \%)\end{array}$ & $\begin{array}{c}106(88.3 \%) \\
13(10.8 \%)\end{array}$ & $\begin{array}{c}142(97.2 \%) \\
4(2.8 \%)\end{array}$ \\
\hline
\end{tabular}

primíparas y el $92.7 \%$ de mujeres españolas. En el grupo de gestantes con distinta nacionalidad, las de origen magrebí constituyeron el colectivo más numeroso.

Con relación al consumo de tabaco, en la muestra estudiada hemos encontrado 152 gestantes que se declararon fumadoras $(33.7 \%)$, de las que 68 mantuvieron el consumo de tabaco durante el embarazo, por lo que la prevalencia global del consumo de tabaco en las gestantes resultó ser del $15.07 \%$. En la muestra estudiada el consumo de tabaco fue menor al final del embarazo, de forma que la prevalencia en el grupo de gestantes de tercer trimestre disminuyó significativamente con relación a las del segundo y primer trimestre $\left(\chi^{2}=13.114\right.$ y $18.715, \mathrm{p}<0.0003$ y p $<0.0001$, respectivamente). La distribución por trimestres queda reflejada en la TABLA 2.

Un total de 123 gestantes reconocieron consumir o haber consumido alcohol durante el embarazo, lo que supone una prevalencia de exposición global durante el embarazo del $27.2 \%$, siendo el consumo medio mensual de 16.5 UBE (DT 20.5). El consumo resultó ser significativamente mayor $\left(\chi^{2}=9.48 \mathrm{p}<0.002\right)$ en el grupo de gestantes de primer trimestre $(40.7 \%)$, y progresivamente menor en el resto de trimestres $(23.1 \%$ en el grupo de segundo trimestre; y $17.1 \%$ en el tercer trimestre). En el grupo de gestantes expuestas, el $52.8 \%$ declaró un consumo de 1 o 2 UBE semanales, el $45.9 \%$ entre 2 y 10 UBE, y un $3.1 \%$ reconoció consumos semanales superiores a las 10 UBE.

El tipo de bebida más frecuentemente consumida correspondió a la cerveza ( $68.8 \%$ de las consumidoras), seguida de los destilados (26.9\% de las consumidoras) y del vino (20.8\% de las consumidoras). El número de consumidoras

Tabla 2

Exposición al tabaco durante el embarazo

\begin{tabular}{lccc}
\hline Tabaco & $\begin{array}{c}\mathbf{1}^{\text {er }} \text { Trimestre } \\
\text { (N = 184) }\end{array}$ & $\begin{array}{c}\mathbf{2}^{\mathbf{0}} \text { Trimestre } \\
\text { (N = 124) }\end{array}$ & $\begin{array}{c}\mathbf{3}^{\mathrm{ep}} \text { Trimestre } \\
\text { (N = 146) }\end{array}$ \\
\hline $\begin{array}{l}\text { Edad Inicio } \\
\text { Edad en años }\end{array}$ & $16.7(\mathrm{DT} 2.9)$ & $17.2(\mathrm{DT} 3.3)$ & $17(\mathrm{DT} 2.7)$ \\
\hline $\begin{array}{l}\text { Antes del embarazo } \\
\text { Sí fumaba }\end{array}$ & $67(36 \%)$ & $37(29.6 \%)$ & $48(35.7 \%)$ \\
No fumaba & $117(63.6 \%)$ & $87(69.6 \%)$ & $98(64.3 \%)$ \\
\hline $\begin{array}{l}\text { En el embarazo } \\
\text { Sí fuma }\end{array}$ & $39(21.2 \%)$ & $23(18.5 \%)$ & $6(13.3 \%)$ \\
No fuma & $145(78.8 \%)$ & $101(81.5 \%)$ & $140(86.7 \%)$ \\
\hline $\mathbf{N}^{0}$ de cigarrillos & & & \\
Antes & $13.0(\mathrm{DT} 6.2)$ & $14.3(\mathrm{DT} 7.7)$ & $14.8(\mathrm{DT} 9.0)$ \\
Durante & $4.9(\mathrm{DT} 3.3)$ & $6.3(\mathrm{DT} 4.4)$ & $7.9(\mathrm{DT} 8.0)$ \\
\hline Su pareja fuma & & & \\
Sí & $60(32.6 \%)$ & $32(26 \%)$ & $34(34.3 \%)$ \\
No & $116(63 \%)$ & $89(73 \%)$ & $64(64.6 \%)$ \\
\hline Exposición Pasiva & & & \\
Casa & $39(21.3 \%)$ & $18(15.1 \%)$ & $14(14.7 \%)$ \\
Trabajo & $9(4.9 \%)$ & $7(6 \%)$ & $4(4.2 \%)$ \\
\hline
\end{tabular}


se incrementaba significativamente, en las gestantes de primer y segundo trimestre, durante los fines de semana $\left(\chi^{2}\right.$ $=9.8$ y $9.56, \mathrm{p}<0.0017$ y $\mathrm{p}<0.002$ respectivamente $)$, aumentando un $29.1 \%$ respecto a los días de diario. El $11.3 \%$ de las gestantes que consumían alcohol lo hacían a diario, el $35.3 \%$ semanalmente, y el $54.3 \%$ con una cadencia mensual. En la TABLA 3 quedan recogidos los principales resultados según el trimestre de embarazo.

Con relación al consumo de sustancias ilícitas (TABLA 4), 11 pacientes reconocieron haber consumido cannabis durante el embarazo, lo que supone una prevalencia del consumo del $2.43 \%$. Ninguna gestante reconoció el consumo de otro tipo de droga ilícita.

No hemos observado diferencias en el consumo de tabaco, alcohol, u otras drogas en ninguno de los trimestres con relación a la edad de las gestantes, paridad, situación laboral, nacionalidad o distrito de residencia. Hemos observado asociación significativa entre el nivel de estudios y el consumo de tabaco y alcohol, globalmente y por trimestres. Cabe señalar que el consumo de tabaco resultó menor en las ges-

Tabla 3

Exposición al alcohol durante el embarazo

\begin{tabular}{|c|c|c|c|}
\hline Alcohol & $\begin{array}{c}1^{\text {er }} \text { Trimestre } \\
\text { ( } N=184)\end{array}$ & $\begin{array}{c}2^{\circ} \text { Trimestre } \\
(\mathrm{N}=121)\end{array}$ & $\begin{array}{c}3^{\varepsilon \rho} \text { Trimestre } \\
(N=146)\end{array}$ \\
\hline \multicolumn{4}{|l|}{ Consumo } \\
\hline Sí & 75 (40.7\%) & 28 (23.1\%) & $25(17.1 \%)$ \\
\hline No & 109 (59.3\%) & $93(76.5 \%)$ & $121(82.9 \%)$ \\
\hline \multicolumn{4}{|c|}{ Tipo en laborables } \\
\hline Cerveza & 40 (57\%) & 11(39.3\%) & $18(70 \%)$ \\
\hline Vino & $20(28.6 \%)$ & $11(39.3 \%)$ & $7(25 \%)$ \\
\hline Licores & $13118.6 \%$ & $2(7.1 \%)$ & $1(5 \%)$ \\
\hline \multicolumn{4}{|c|}{ Tipo en festivos } \\
\hline Cerveza & $47(67.1 \%)$ & 20 (71.4\%) & $17(68 \%)$ \\
\hline Vino & $29(41.4 \%)$ & 14 (50\%) & $7(28 \%)$ \\
\hline Licores & $27(38.6 \%)$ & $4(14.3 \%)$ & $7(28 \%)$ \\
\hline \multicolumn{4}{|c|}{ Frec. de consumo } \\
\hline Diario & $9(12.8 \%)$ & $2(7.1 \%)$ & $3(12 \%)$ \\
\hline Semanal & $24(34.2 \%)$ & $10(35.7 \%)$ & $9(36 \%)$ \\
\hline Mensual & 40 (57.1\%) & 14 (50\%) & $14(56 \%)$ \\
\hline \multicolumn{4}{|c|}{ UBE semanal } \\
\hline$<2$ UBE & $36(51.4 \%)$ & $18(64.2 \%)$ & $11(55 \%)$ \\
\hline 2-10 UBE & $28(40 \%)$ & $9(32.1 \%)$ & $9(32.1 \%)$ \\
\hline$>10$ UBE & $6(8.6 \%)$ & $1(5 \%)$ & \\
\hline \multicolumn{4}{|c|}{ UBE semanal } \\
\hline Promedio & 16.7 (DT 20.6) & 8.5 (DT 11.1) & 12 (DT 9.9) \\
\hline
\end{tabular}

Tabla 4

Exposición a cannabis durante el embarazo

\begin{tabular}{lccc}
\hline Drogas ilícitas & $\begin{array}{c}\mathbf{1}^{\text {er }} \text { Trimestre } \\
(\mathbf{N}=\mathbf{1 8 4})\end{array}$ & $\begin{array}{c}\mathbf{2}^{\mathbf{0}} \text { Trimestre } \\
(\mathbf{N}=\mathbf{1 2 1})\end{array}$ & $\begin{array}{c}\mathbf{3}^{\mathrm{e}} \text { Trimestre } \\
\mathbf{( N = 1 4 6 )}\end{array}$ \\
\hline Consumo Marihuana & & & \\
Sí & $7(4.8 \%)$ & $2(1.9 \%)$ & $2(1.2 \%)$ \\
No & $137(85.2 \%)$ & $103(98.1 \%)$ & $83(98.8 \%)$ \\
\hline
\end{tabular}

Tabla 5

Asociación de nivel alto de estudios y tabaquismo de la pareja en el consumo de tabaco y alcohol. (RR: Riesgo Relativo)

\begin{tabular}{|c|c|c|c|}
\hline & Primer T & Segundo T & Tercer T \\
\hline \multicolumn{4}{|c|}{ Nivel alto de estudios } \\
\hline \multicolumn{4}{|c|}{ Consumo de Tabaco } \\
\hline $\begin{array}{l}\text { RR } \\
\text { IC95\% } \\
\text { Signif. }\end{array}$ & $\begin{array}{c}0.341 \\
(0.187-0.623) \\
p<0.0001\end{array}$ & $\begin{array}{c}0.455 \\
(0.206-1.00) \\
p<0.039\end{array}$ & $\begin{array}{c}0.778 \\
(0.345-1.756) \\
p<0.39 \text { NS }\end{array}$ \\
\hline \multicolumn{4}{|c|}{ Consumo de Alcohol } \\
\hline $\begin{array}{l}\text { RR } \\
\text { IC } 95 \% \\
\text { Signif. }\end{array}$ & $\begin{array}{c}2.455 \\
(1.298-4.641) \\
p<0.004\end{array}$ & $\begin{array}{c}4.091 \\
(1.134-14.759) \\
p<0.017\end{array}$ & $\begin{array}{c}3.143 \\
(0.95-10.21) \\
p<0.042\end{array}$ \\
\hline \multicolumn{4}{|c|}{ Tabaquismo en la pareja } \\
\hline \multicolumn{4}{|c|}{ Consumo de Tabaco } \\
\hline $\begin{array}{l}\text { RR } \\
\text { IC95\% } \\
\text { Signif. }\end{array}$ & $\begin{array}{c}17.208 \\
7.648-38.721 \\
p<0.0001\end{array}$ & $\begin{array}{c}24.364 \\
(7.749-76.606) \\
p<0.0001\end{array}$ & $\begin{array}{c}5.576 \\
(2.272-13.685) \\
p<0.0001\end{array}$ \\
\hline
\end{tabular}

tantes con mayor nivel de estudios (RR 0.659 [0.537-0.810] $\mathrm{p}<0.0001)$, mientras que el consumo de alcohol resultó más frecuente en estas gestantes con mayor nivel estudios (RR 1.87 [1.30-2.69] $\mathrm{p}<0.0007)$. En la TABLA 5 se recogen los valores de RR para cada uno de los trimestres.

Hemos observado una asociación significativa entre el consumo de tabaco durante el embarazo y el tabaquismo de la pareja, con RR 3.091 [2.501-3.820] p< 0.0001. Además, el consumo de alcohol durante el primer trimestre resultó estar asociado al consumo de tabaco (RR 2.357 [1.243-4.469] $\mathrm{p}<0.006)$. Esta asociación no fue significativa en el segundo o tercer trimestre.

\section{Discusión}

El presente trabajo responde a un diseño observacional de corte transversal sobre el consumo de tóxicos durante el embarazo. Las limitaciones que plantea un estudio basado en la autodeclaración de las gestantes ha sido puesta de manifiesto en estudios previos (Aranda, Mateos, González, Sánchez y Luna, 2008). Nuestro estudio, al utilizar un cuestionario autoadministrado anónimo que no permitía trazabilidad de las respuestas, y que la gestante introducía personalmente en una caja precintada le proporcionaba garantías de confidencialidad, aumentando la fiabilidad de las respuestas. Por otro lado, aunque al interpretar los resultados es preciso tener en cuenta la posible infradeclaración (Castellanos et al., 2000), los resultados obtenidos, sobre todo en relación al consumo de alcohol, son suficientemente llamativos como para alertar a los proveedores de atención obstétrica sobre la necesidad de poner en marcha medidas preventivas.

El embarazo parece condicionar cierto efecto modulador sobre el consumo de tabaco, de forma que mientras que la 
proporción de embarazadas de primer trimestre que fumaba con anterioridad al embarazo era del 36\%, tan sólo el $21.2 \%$ continuaba fumando durante el mismo. La proporción de fumadoras en los grupos de segundo y tercer trimestre también era menor, siendo las gestantes que se encontraban en el tercer trimestre las que presentaron una menor tasa de consumo (13.3\%), así como una mayor diferencia entre la proporción de mujeres que fumaba antes del embarazo (35.7\%) y las que continuaban haciéndolo. El estado de sobrecarga física que el tercer trimestre condiciona, junto a las medidas de promoción de la salud que se llevan a cabo desde atención primaria, son los factores que más pueden influir en la tendencia. No obstante, es llamativo que el consumo declarado de tabaco durante el primer trimestre alcanza cifras similares a las descritas en gestantes de hace 30 años (Herrera, 1989). Las campañas de información desarrolladas en los últimos años han tenido un impacto limitado en la reducción del consumo, sobre todo en las fases iniciales del embarazo. De hecho, aunque según diversos estudios existe un patrón de descenso de la prevalencia de fumadoras habituales entre las jóvenes españolas, la incorporación al consumo es llamativamente más precoz y numerosa en el grupo de mujeres que en el de varones de la misma edad (Villalbí et al., 2012), hecho que podría estar en relación con estrategias de promoción de las multinacionales tabaqueras o con aspectos relacionados con la identidad de género (Amos y Bostock, 2007). Aunque posteriormente las encuestas de salud señalan que la prevalencia es similar en ambos sexos, o ligeramente mayor entre los varones, el número de fumadoras en la edad reproductiva es muy elevado, por lo que la proporción de mujeres fumadoras que quedan embarazadas alcanza cifras del $36 \%$ en nuestro estudio.

Por otro lado, hemos observado un consumo de alcohol significativamente mayor en el grupo de gestantes con mayor cualificación académica en el primer y segundo trimestre. La existencia de gradientes sociales, educacionales y ocupacionales en el consumo de alcohol ha sido puesta de manifiesto con anterioridad en estudios realizados sobre la población general femenina, así, en la Encuesta Nacional de Salud 2011-2012, queda reflejado que mientras el 52.05\% de las mujeres entre 25 y 64 años con estudios superiores habían consumido alcohol en las 2 semanas previas a la encuesta, sólo el $33.86 \%$ de las mujeres con menor nivel de estudios lo había hecho. Por otro lado, el $71.7 \%$ de las mujeres universitarias había bebido alcohol en el último año, frente al $27.9 \%$ de las mujeres sin estudios, manteniéndose la diferencia en todos los grupos de edad (Instituto Nacional de Estadística, 2013).

Resulta llamativo que el grupo de embarazadas con mayor nivel cultural, y, por tanto, con más recursos para acceder a la información referente a los potenciales efectos del consumo de bebidas alcohólicas durante el embarazo, presente una prevalencia mayor. Posiblemente los hábitos so- ciales y laborales de estas mujeres difieran de los que presentan mujeres con menor nivel de formación, condicionando diferencias en el consumo. Aunque en la literatura internacional no exista acuerdo sobre la existencia de cantidades tolerables de ingesta alcohólica durante el embarazo, las autoridades sanitarias de nuestro país aconsejan la ausencia de consumo. De hecho, el Ministerio español de Sanidad, Política Social e Igualdad (2010) utiliza en su estrategia para evitar el consumo de alcohol durante el embarazo el lema "Embarazadas: cero alcohol", o "Si estás embarazada, con el alcohol no hay excusa que valga". Aunque realmente la información de estos programas de prevención llega a las gestantes, ya que el consumo de alcohol disminuye a lo largo del embarazo, sería importante encontrar mejores vías de comunicación con los grupos de mayor vulnerabilidad. La sistematización de la asistencia obstétrica con visitas mensuales a los centros de salud o a los hospitales para el control del embarazo, supone una oportunidad para ofrecer a las gestantes la información referente a la necesidad de evitar el consumo de alcohol, siendo a nuestro juicio, una vía idónea de comunicación con la población susceptible. La consulta de consejo pre-concepcional y la primera visita de control obstétrico son marcos idóneos para comunicar de forma inequívoca la necesidad de evitar el consumo de tóxicos, poniendo en marcha estrategias educativas específicas integradas en los programas de educación maternal.

La eficacia de la intervención educativa en la reducción del consumo durante el embarazo, ha sido evaluada en diversos estudios (Chang et al., 1999; O'Connor, 2007; Reynolds et al., 1995) que, aunque con resultados dispares, sugieren que las intervenciones psicológicas y educacionales pueden ayudar a reducir el consumo de alcohol o mantener las mujeres abstinentes durante el embarazo. Para ello es necesario que el personal sanitario implicado en la asistencia cuente con la motivación y preparación necesarias. En nuestro entorno no se ha evaluado el efecto de dichas intervenciones en el consumo de alcohol durante el embarazo, ni su nivel de desarrollo en las diferentes comunidades autónomas.

El primer trimestre de la gestación, al menos su primera mitad, pasa desapercibido para muchas de las embarazadas, sobre todo cuando se trata de gestaciones no planificadas. Esto condiciona que el consumo de alcohol durante el primer trimestre alcance una prevalencia en nuestro estudio del $40.7 \%$, en consonancia con datos publicados en ciudades españolas de parecido perfil, como es el caso de Barcelona (García-Algar et al., 2008). En este sentido, cabe señalar que el consumo de alcohol también se relaciona con la existencia de conductas sexuales de riesgo para embarazos no planificados, como recientemente han puesto de manifiesto en nuestro país Espada, Morales, Orgilés, Piqueras y Carballo (2013), señalando que el uso de preservativos es 1.4 veces menos frecuente entre los adolescentes que practican sexo bajo la influencia del alcohol. 
Como habíamos señalado anteriormente, el propio embarazo podría modular el consumo, de forma que la proporción de consumidoras de alcohol es menor en los grupos de gestantes en segundo y tercer trimestre. A pesar de todo, niveles de consumo del $23.1 \%$ y del $17 \%$ en el segundo y tercer trimestre respectivamente son excesivamente elevados si consideramos los objetivos planteados en 2010 por el Ministerio de Sanidad sobre la abstinencia alcohólica durante el embarazo (Ministerio de Sanidad, Política Social e Igualdad, 2010).

Aunque más de la mitad de las gestantes reconocen patrones de consumo esporádico (mensual), existe un $12.8 \%$ de embarazadas en el primer trimestre, un $7 \%$ en el segundo y un $12 \%$ en el tercero que reconoce consumir alcohol diariamente. Por otro lado, aunque algo más de la mitad de las gestantes reconoce un consumo semanal inferior a las 2 UBE, más de la tercera parte declara mantener un consumo semanal de hasta 10 UBE. Cabe señalar, que no existe un consenso claro sobre la cantidad de alcohol que puede considerarse inocua, si bien, sus potenciales efectos sobre el feto, tanto en resultados perinatales (prematuridad, bajo peso, o síndrome alcohólico fetal) (Patra et al., 2011) como a más largo plazo en lo referente a los trastornos del espectro alcohólico fetal (López y Arán, 2010) determinan que el consumo de alcohol durante el embarazo sea totalmente desaconsejado (Grupo de Trabajo de la Guía de Práctica Clínica de Atención en el Embarazo y Puerperio, 2014; Ministerio de Sanidad, Política Social e Igualdad, 2010; Poli et al., 2013). La OMS pone de manifiesto las ventajas de aconsejar la completa abstinencia durante el embarazo así como de desarrollar medidas de intervención en las gestantes consumidoras.

Hemos encontrado un $2.43 \%$ de gestantes que reconocían el consumo de cannabis, si bien, ninguna de la embarazadas reconoció consumir otras drogas ilegales. El Estudio Colaborativo Español sobre Malformaciones Congénitas 1976-1996 puso de manifiesto la existencia de variaciones en el consumo de drogas en función del grupo étnico de la embarazada, oscilando entre el $5.5 \%$ en las embarazadas de etnia gitana y un $1.1 \%$ en la gestantes blanca caucásicas (Martínez-Frías, 1999). Posiblemente las posibles connotaciones legales y sociosanitarias asociadas al consumo de drogas ilícitas fueron obstáculos para que las gestantes incluidas en nuestro estudio reconocieran consumos diferentes del cannabis. Sin embargo, la morbilidad fetal y neonatal asociada al consumo de estas sustancias pone de manifiesto la necesidad de incluir información referente a estas sustancias en las medidas de intervención educativa durante el embarazo, con objeto de reducir el consumo.

Son diversos los aspectos que limitan la interpretación de los resultados de nuestro estudio. El uso de un cuestionario autoadministrado supone un potencial factor de sesgo derivado de la infra-declaración de las pacientes. Además, no se han realizado pruebas de laboratorio que permitan corro- borar objetivamente los niveles de consumo encontrados, por lo que probablemente los niveles reales puedan ser incluso superiores a los declarados por las pacientes. Por otro lado, la intención de evaluar simultáneamente el consumo de tabaco, alcohol y otras drogas, y su intensidad, nos llevó a utilizar un único cuestionario, elaborado con preguntas ya validadas anteriormente, si bien el cuestionario en su conjunto no ha sido sometido a las pruebas de validez pertinentes. Finalmente, al tratarse de un estudio transversal obtenemos datos sobre prevalencia del consumo de tóxicos y su intensidad, no siendo posible evaluar la progresión del consumo durante los diferentes trimestres.

No obstante, los resultados obtenidos son suficientemente concluyentes para afirmar que las campañas de educación sanitaria dirigidas a evitar el consumo de tóxicos durante el embarazo deben intensificar esfuerzos, suministrando información adecuada a las gestantes e incorporando estrategias educativas específicas que abarquen desde el período pre-concepcional a la lactancia. El desarrollo del consejo pre-gestacional, y el diseño de programas de formación para gestantes, con sesiones encaminadas a conseguir la abstinencia de consumo durante el embarazo (y la lactancia) se plantean como estrategias necesarias en nuestro entorno. En la puesta en marcha de estas medidas, el papel de los profesionales sanitarios, tanto de atención primaria como de atención especializada, será fundamental para la difusión de mensajes inequívocos.

\section{Conflicto de intereses}

Los autores declaran no tener ningún conflicto de interés.

\section{Referencias}

Amos, A., y Bostock, Y. (2007). Young people, smoking and gender - a qualitative exploration. Health Educational Research, 22, 770-781. doi: 10.1093/her/cyl075.

Aranda, J. M., Mateos, P., González, A., Sánchez, F., y Luna, J. D. (2008). Validez de distintas medidas de consumo de tabaco durante el embarazo: especificidad, sensibilidad y puntos de corte dónde y cuándo. Revista Española de Salud Pública, 82, 535-545.

Babor, T. F., Higgins-Biddle, J. C., Saunders, J. B., y Monteiro, M. G. (2001). Cuestionario de Identificación de los Transtornos debidos al Consumo de Alcohol. Ginebra: Organización Mundial de la Salud. Recuperado de http:// www.who.int/substance_abuse/activities/en/AUDITmanualSpanish.pdf

Bernstein, I. M., Plociennik, K., Stahle, S., Badger, G. J., y Secker-Walker, R. (2000). Impact of maternal cigarette smoking on fetal growth and body composition. American Journal Obstetrics and Gynecology, 183, 883-886. doi: $10.1067 /$ mob.2000.109103 
Bouras, G., Theofanopoulou, N., Mexi-Bourna, P., Poulios, A., Michopoulos, I., Tassiopoulou, I.,... Christodoulou, C. (2013). Preterm birth and maternal psychological health. Journal of Health Psychology, 1359105313512353. doi: $10.1177 / 1359105313512353$.

Castellanos, M. E., Muñoz, M. I., Nebot, M., Payá, A., Rovira, M. T., Planasa, S.,... Carreras, R. (2000). Validez del consumo declarado de tabaco en el embarazo. Atención Primaria, 9, 629-632. doi: 10.1016/S0212$6567(00) 78736-1$.

Chang, G., Wilkins-Haug, L., Berman, S. y Goetz, M. A. (1999). Brief intervention for alcohol use in pregnancy: randomized trial. Addiction, 94,1499-1508. doi: 10.1046/j.1360-0443.1999.941014996.x.

Cnattingius, S. (2004). The epidemiology of smoking during pregnancy: smoking prevalence, maternal characteristics and pregnancy outcomes. Nicotine and Tobacco Research, 6, 125-140. doi: 10.1080/14622200410001669187.

Comité Nacional para la Prevención del Tabaquismo (CNPT). (2007). Libro blanco sobre mujeres y tabaco. Madrid: Ministerio de Sanidad y Consumo. Recuperado de http://www.cnpt.es/doc_pdf/mujerestabaquismo.pdf

Delegación del Gobierno para el Plan Nacional Sobre Drogas. (2011). Plan Nacional Sobre Drogas. Memoria del Ministerio de Sanidad, Servicios Sociales e Igualdad. Madrid: Ministerio de Sanidad, Servicios Sociales e Igualdad. Recuperado de http://www.pnsd.msc.es/Categoria2/ publica/pdf/oed-2007.pdf.

Doz-Mora, J. F., Gasulla-Pascual, G., Cárceles-Jurado, S., Guerrero-Bartolín, I., Tintó-Padró, A., Prat-Asensio, C., y Rosell-Murphy, M. (2004). Evolución del hábito tabáquico durante el embarazo y el posparto. Atención Primaria, 34, 465-471.

Espada, J. P., Morales, A., Orgilés, M., Piqueras, J. A., y Carballo, J. L. (2013). Comportamiento sexual bajo la influencia del alcohol en adolescentes españoles. Adicciones, 25, 55-62.

Eurocare. (2011). Protecting the unborn baby from alcohol, policy debate. Bruselas: European Alcohol Policy Alliance. Recuperado de http://www.eurocare.org/resources/ policy_issues/alcohol_and_pregnancy

Eurocat. (2014). European surveillance of congenital anomalies prevalence tables. Derry: University of Ulster. Recuperado de http://www.eurocat-network.eu/ACCESSPREVALENCEDATA/PrevalenceTables

García-Algar, O., Kulaga, V., Gareri, J., Koren, G., Vall, O., Zuccaro, P.,... Pichini S. (2008). Alarming prevalence of fetal alcohol exposure in a Mediterranean city. Therapeutic Drug Monitoring, 30, 249-254.

Garcia-Algar, O., Vall Combelles, O., Puig Sola, C., Mur Sierra, A., Scaravelli, G., Pacifici, R., y Pichini, S. (2009). Exposición prenatal a drogas de abuso a través del análisis de meconio en una población de bajo nivel socioeconó- mico en Barcelona. Anales de Pediatría, 70, 151-158. doi: 10.1097/FTD.0b013e31816a8657.

Genbacev, O., Blass, K. E., Joslin, S. J., y Fisher, S. J. (1995). Maternal smoking inhibts early human cytothrofoblast differentiation. Reproductive Toxicology, 9, 245-255.

Gómez, A., Conde, A., y Aguiar, J. A. (2001). Utilidad Diagnóstica del cuestionario Alcohol Use Disorders Identification Test (AUDIT) para detectar el consumo de alcohol de riesgo en atención primaria. Medicina Clínica, $116,121-124$.

Grupo de trabajo de la Guía de Práctica Clínica de Atención en el Embarazo y Puerperio. (2014). Guía de práctica clínica de atención en el embarazo y puerperio. Madrid: Ministerio de Sanidad, Servicios Sociales e Igualdad. Recuperado de http://www.juntadeandalucia.es/ salud/servicios/contenidos/nuevaaetsa/up/AETSA_2011_10_GPC_Embarazo.pdf

Herrera, J. (1989). El tabaco y su relación con aspectos perinatales. (Tesis doctoral no publicada). Madrid: Universidad Complutense de Madrid.

Instituto Nacional de Estadística. (2013). Encuesta Nacional de salud 2011-2012. Madrid: Instituto Nacional de Estadística. Recuperado de http://www.ine.es/jaxi/menu. do?type $=$ pcaxis\&path=/t15/p419/a2011/p06/\&file=pcaxis

Llopis, J. J., Gual, A., y Rodríguez-Martos A. (2000). Registro del consumo de bebidas alcohólicas mediante la unidad de bebida estándar. Diferencias geográficas. Adicciones, 12, 11-19.

Lopez, M. B., y Arán, V. (2010). Perfiles ejecutivos en niños y adolescentes con trastornos del espectro fetal alcohólico. Acta Psiquiátrica y Psicológica de América Latina, 56, 294-304.

Martínez-Frías, M. L. (1999). Análisis del riesgo de defectos congénitos debidos a la ingesta de drogas durante el embarazo. Medicina Clínica, 112, 41-44.

Martínez-Frías, M. L., Rodríguez-Pinilla, E., y Bermejo, E. (2005). Consumo de tabaco durante el embarazo en España: análisis por años, comunidades autónomas y características maternas. Medicina Clínica, 124, 86-92.

Ministerio de Sanidad, Política Social e Igualdad. (2010). Embarazadas cero alcohol. Madrid: Ministerio de Sanidad, Servicios Sociales e Igualdad. Recuperado de http:// www.embarazadasceroalcohol.es/embarazadas_cero_alcohol.htlm

Ministerio de Sanidad, Servicios Sociales e Igualdad. (2013). Encuesta Nacional de Salud. España 2011/12. Serie informes monográficos $n^{\circ} 1$. Consumo de alcohol. Madrid: Ministerio de Sanidad, Servicios Sociales e Igualdad. Recuperado de http://www.pnsd.msc.es

O'Connor, M., y Whaley, S. (2007). Brief intervention for alcohol use by pregnant women. American Journal of Public Health, 97, 252-258. doi: 10.2105/AJPH.2005.077222 
Patra, J., Bakker, R., Irving, H., Jaddoe, V. W., Malini, S., y Rehm J. (2011). Dose-response relationship between alcohol consumption before and during pregnancy and the risks of low birthweight, preterm birth and small for gestational age (SGA) - a systematic review and meta-analyses. British Journal Obstetrics and Gynecology, 118, 1411-1421. doi: 10.1111/j.1471-0528.2011.03050.x

Pichini, S., Puig, C., García-Algar, O., Pacifici, R., Figueroa, C., Vall, O.,... Sunyer, J. (2002). Efectos neonatales del hábito tabáquico durante el embarazo y determinantes sociodemográficos en Barcelona. Medicina Clínica, 118, 53-56.

Poli, A., Marangoni, F., Avogaro, A., Barba, G., Bellentani, S., Bucci, M.,... Visioli, F. (2013). Moderate alcohol use and health: A consensus document. Nutrition, Metabolism and Cardiovascular Diseases, 23, 487-504. doi: 10.1016/j. numecd.2013.02.007.

Reynolds, K. D., Coombs, D. W., Lowe, J. B., Peterson, P. L., y Gayoso, E. (1995). Evaluation of a self-help program to reduce alcohol consumption among pregnant women. International Journal of the Addictions, 30(4), 499-508. doi: $10.3109 / 10826089509048735$.

Toothily, D. P., Stewart, J. H., Coles, E. C., Andrews, J., y Cartlidge, P. H. (1999). Maternal cigarette smoking and pregnancy outcome. Paediatric Perinatal Epidemiology, 13ED, 245-53. doi: 10.1046/j.1365-3016.1999.00187.x.

Villalbí, J. R., Suelves, J. M., García-Continente, X., Saltó, E., Ariza, C., y Cabezas, C. (2012). Cambios en la prevalencia del tabaquismo en los adolescentes en España. Atención Primaria, 44, 36-42. 


\section{DATOS DEMOGRÁFICOS}

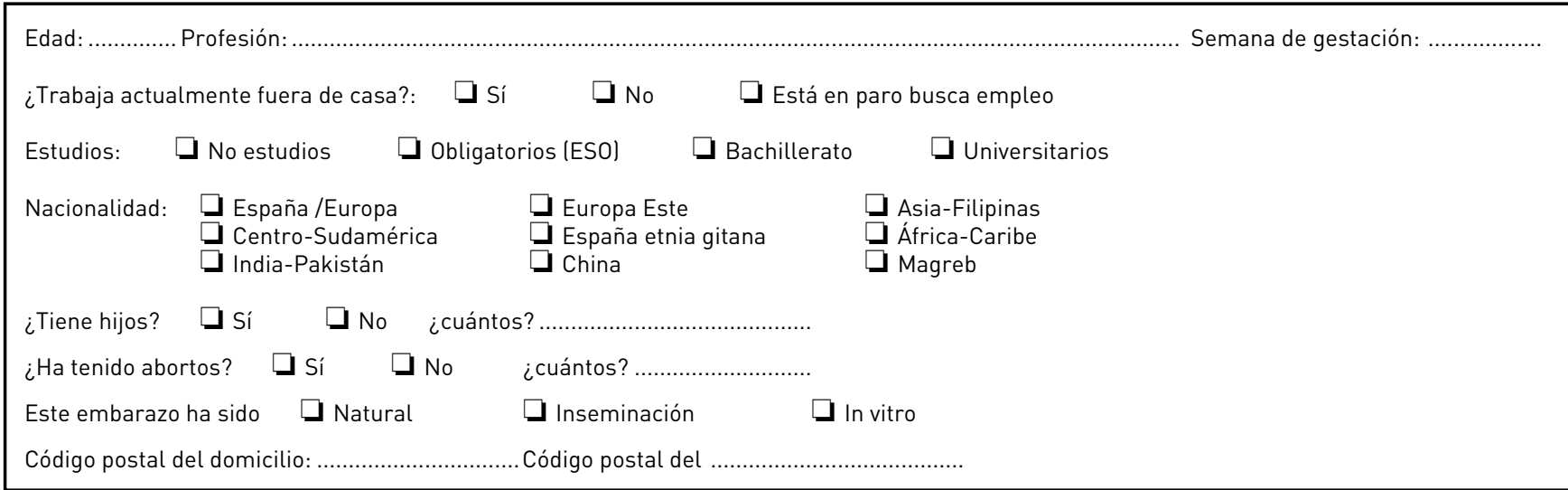

\section{TABACO}

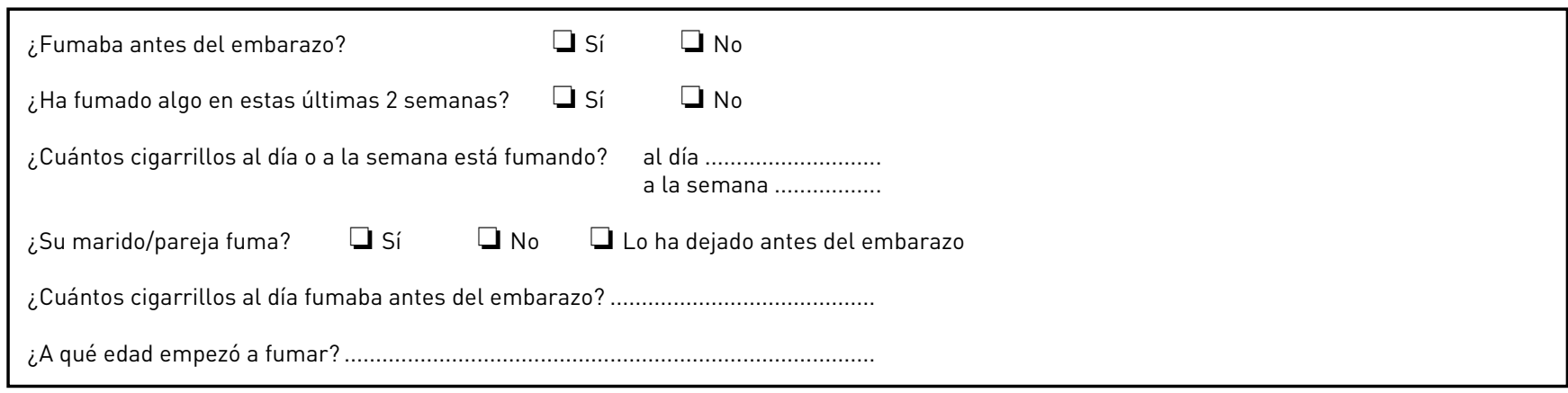

\section{ALCOHOL}

De Lunes a viernes. Indique con qué frecuencia consume bebidas alcohólicas entre semana. Especifique también el tipo de bebida.

\begin{tabular}{|c|c|c|c|c|}
\hline & $\begin{array}{l}\text { RECIPIENTE } \\
\text { (elegir uno) }\end{array}$ & $\begin{array}{c}\text { DIARIAMENTE } \\
\text { Número de veces: } 0,1,2, \ldots\end{array}$ & $\begin{array}{c}\text { SEMANALMENTE } \\
\text { Número de veces: } 0,1,2, \ldots\end{array}$ & $\begin{array}{c}\text { MENSUALMENTE } \\
\text { Número de veces: } 0,1,2, \ldots\end{array}$ \\
\hline CERVEZA & $\begin{array}{l}\text { Vaso, lata, jarra, } \\
\text { botella mediana, quinto }\end{array}$ & & & \\
\hline VINO & Vaso, copa, botella & & & \\
\hline LICORES & $\begin{array}{l}\text { Chupito, copa, } \\
\text { combinados Licores }\end{array}$ & & & \\
\hline
\end{tabular}

En sábado, domingos y festivos. Indique con qué frecuencia consume bebidas alcohólicas el fin de semana. Especifique también el tipo de bebida.

\begin{tabular}{|c|c|c|c|c|}
\hline & $\begin{array}{l}\text { RECIPIENTE } \\
\text { (elegir uno) }\end{array}$ & $\begin{array}{c}\text { DIARIAMENTE } \\
\text { Número de veces: } 0,1,2, \ldots\end{array}$ & $\begin{array}{c}\text { SEMANALMENTE } \\
\text { Número de veces: } 0,1,2, \ldots\end{array}$ & $\begin{array}{c}\text { MENSUALMENTE } \\
\text { Número de veces: } 0,1,2, \ldots\end{array}$ \\
\hline CERVEZA & $\begin{array}{l}\text { Vaso, lata, jarra, } \\
\text { botella mediana, quinto }\end{array}$ & & & \\
\hline VINO & Vaso, copa, botella & & & \\
\hline
\end{tabular}

\section{OTRAS SUSTANCIAS}

Indique si consume alguna sustancia como las que se describen a continuación

$\begin{array}{llccc}\text { MARIHUANA } & \square \text { DIARIO } & \square \text { SEMANAL } & \square \text { OCASIONAL } & \square \text { NUNCA } \\ \text { COCAÍNA } & \square \text { DIARIO } & \square \text { SEMANAL } & \square \text { OCASIONAL } & \square \text { NUNCA } \\ \text { HEROÍNA } & \square \text { DIARIO } & \square \text { SEMANAL } & \square \text { OCASIONAL } & \square \text { NUNCA } \\ \text { METADONA } & \square \text { DIARIO } & \square \text { SEMANAL } & \square \text { OCASIONAL } & \square \text { NUNCA } \\ \text { ANFETAMINAS } & \square \text { DIARIO } & \square \text { SEMANAL } & \square \text { OCASIONAL } & \square \text { NUNCA } \\ \text { EXTASIS } & \square \text { DIARIO } & \square \text { SEMANAL } & \square \text { OCASIONAL } & \square \text { NUNCA }\end{array}$

